\title{
Conditional Bicistronic Cre Reporter Line Expressing Both Firefly Luciferase and $\beta$-galactosidase
}

\author{
Tomo-o Ishikawa, Harvey R. Herschman
}

Department of Molecular and Medical Pharmacology and Department of Biological Chemistry, Molecular Biology Institute, David Geffen School of Medicine, UCLA, 341 Boyer Hall, 611 Charles E. Young Drive East, Los Angeles, CA 90095, USA

\begin{abstract}
Purpose: The Cre-loxP system has become an important strategy for conditional gene deletion and conditional gene expression in genetically engineered mice. To evaluate Cre recombinase expression, we generated reporter mice that permit both noninvasive imaging in living animals and either ex vivo histochemical/immunohistochemical tissue transgene expression analysis or quantitative enzyme analysis in the same animal.

Procedures: Transgenic reporter mice were generated in which a loxP-flanked enhanced green fluorescent protein (EGFP) reporter gene and STOP sequence are placed after the nearly ubiquitously expressed CAG promoter, but before a bicistronic transcriptional unit containing luciferase and $\beta$-galactosidase reporter gene coding sequences.

Results: After global deletion of the floxed STOP sequence by germ line Cre deletion, the reporter mouse expresses luciferase and $\beta$-galactosidase in all tissues examined. Tissuespecific expression of both reporter genes occurs in reporter mouse strains expressing Cre in skin (K14 keratin Cre), heart (myosin light chair Cre), or colon (Villin Cre).

Conclusion: The luc-gal ${ }^{\mathrm{Tg}}$ reporter mouse allows noninvasive imaging of target Cre activation both in living animals and in tissues and cells following necropsy, using loss of EGFP expression, gain of luciferase expression, and gain of $\beta$-galactosidase expression as alternatives within the same animal for qualitative analysis of Cre expression.
\end{abstract}

Key words: Cre recombinase, Transgenic mouse, Luciferase, $\beta$-galactosidase, Molecular imaging

\section{Introduction}

$\mathrm{T}$ he Cre-loxP targeted recombination system has been widely used for conditional control of gene expression $[1,2]$. Cre recombinase mediates recombination excision of DNA sequences between its target loxP sites, resulting in the

\footnotetext{
Manuscript significance This manuscript describes a new reporter mouse that can be used to image Cre recombinase activity by noninvasive imaging in living animals, by ex vivo enzyme analysis, and by histochemical and immunohistochemical tissue staining.
}

Correspondence to: Harvey R. Herschman; e-mail: hherschman@mednet. ucla.edu deletion of target sequences in the genome. Appropriate conditional gene alterations using the Cre-loxP system depend on well-characterized and defined Cre expression in targeted cells. Therefore, evaluation of the pattern of Cre expression is important both in achieving the targeted genetic alteration and in avoiding (or at least being aware of) undesired Cre-mediated recombination events. To facilitate the evaluation of Cre-targeted recombination, a number of Cre reporter mouse lines have been developed to determine the pattern of Cre expression in vivo. In these lines, Cre expression patterns can be visualized by reporter genes whose expression is activated only after Cre recombinase-mediated gene remodeling. A variety of reporter genes have been used for this purpose, including enzymes that employ colorimetric 
reactions for histology and in vitro analysis of tissue extracts [3-6], fluorescent proteins [7-14], and enzymes that produce bioluminescent products $[15,16]$.

Recently, molecular imaging has emerged as a powerful method to analyze noninvasively gene expression in animal models [17]. Luciferases have been used as bioluminescent reporter genes whose activities can be monitored in living animals by using sensitive charged-coupled device cameras [18]. There has been extensive and burgeoning use of noninvasive, repeated monitoring of luciferase reporter gene expression in living animals, including the monitoring of Cre expression $[15,16]$. However, one potential disadvantage of luciferase as a reporter gene is the lack of resolution of its expression pattern at the cellular level. It is difficult to obtain reliable expression patterns with the commercially available anti-luciferase antibodies. To address this problem, we prepared a bicistronic construct with the firefly luciferase and $\beta$-galactosidase (lacZ) reporter genes, using an internal ribosomal entry site (IRES), and generated a reporter mouse line, luc-gal ${ }^{\mathrm{Tg}}$, using the strong and widely expressed CAG promoter. In this study, we characterized the bicistronic reporter mouse line by crossing it with both ubiquitous and tissue-specific Cre-expressing murine lines and analyzing luciferase expression in vivo and in necropsy samples and $\beta$-galactosidase expression in necropsy samples.

\section{Materials and Methods}

\section{Generation of Cre Reporter luc-gal ${ }^{\text {Tg }}$ Transgenic Mice}

The vector constructed to generate the Cre reporter mouse (Fig. 1a) includes a CAG promoter [19], loxP sites that flank both the coding region for enhanced green fluorescent protein (EGFP, Clontech, Palo Alto, CA, USA) and a transcriptional/translational STOP sequence (GIBCO, Carlsbad, CA, USA), a transcription unit containing the coding region of firefly luciferase (Promega, Madison, WI, USA), and the coding region of Escherichia coli $\beta$-galactosidase. The two reporter gene coding sequences are linked by the encephalomyocarditis virus (ECMV) IRES (derived from pIRES2-EGFP Clontech). The entire construct is flanked by chicken $\beta$-globin $5^{\prime} \mathrm{HS} 4$ insulator elements $[20,21]$. The insulator element was amplified by polymerase chain reaction from chicken liver genomic DNA by using the following primer sets: HS4F1, GAG CTC ACG GGG ACA GCC CCC CCC CAA ACG; HS4R1, AAT ATT CTC ACT GAC TCC GTC CTG GAG TTG. The plasmid construct was microinjected (transgenic mouse facility, UC Irvine) into the pronuclei of fertilized eggs of $\mathrm{CB} 6 \mathrm{~F} 1$ mice. Founder transgenic mice were selected by genomic Southern blotting and EGFP expression. Tissue EGFP expression was examined by using the Maestro in vivo fluorescence imaging system (CRi Inc., Woburn, MA, USA). For genotyping, the following primer set amplifying $l a c Z$ sequence is used: oIMR0039, ATC CTC TGC ATG GTC AGG TC; oIMR0040, CGT GGC CTG ATT CAT TCC. Mice were also genotyped by observing EGFP expression of either the whole body or tail tip.

\section{Cre-Expressing Mice}

The MlcCre mouse was provided by Dr. Yibin Wang (UCLA). CMVCre (Tg(CMV-cre)1Cgn), K14Cre (Tg(KRT14-cre)1Amc), and VillinCre $(T g($ Vil-cre $) 997 \mathrm{Gum} / J)$ mice were purchased from Jackson Laboratory (Bar Harbor, MA, USA).

\section{Adenovirus}

Ad.CMV.Cre, a nonreplicating adenovirus in which Cre recombinase is under the regulation of the cytomegalovirus (CMV) promoter, was obtained from Dr. Arnold Berk (UCLA). Ad. CMV.Cre $\left(1 \times 10^{9}\right.$ pfu in $100 \mu$ phosphate buffered saline (PBS)) was injected into the tail vein of mice.

\section{In vivo and Ex vivo Imaging of Luciferase Activity}

For in vivo imaging, mice were anesthetized by intraperitoneal administration of a ketamine $(80 \mathrm{mg} / \mathrm{kg}$, Phoenix Pharmaceutical, St. Joseph, MO, USA) and xylazine (4 mg/kg, Phoenix Pharma-
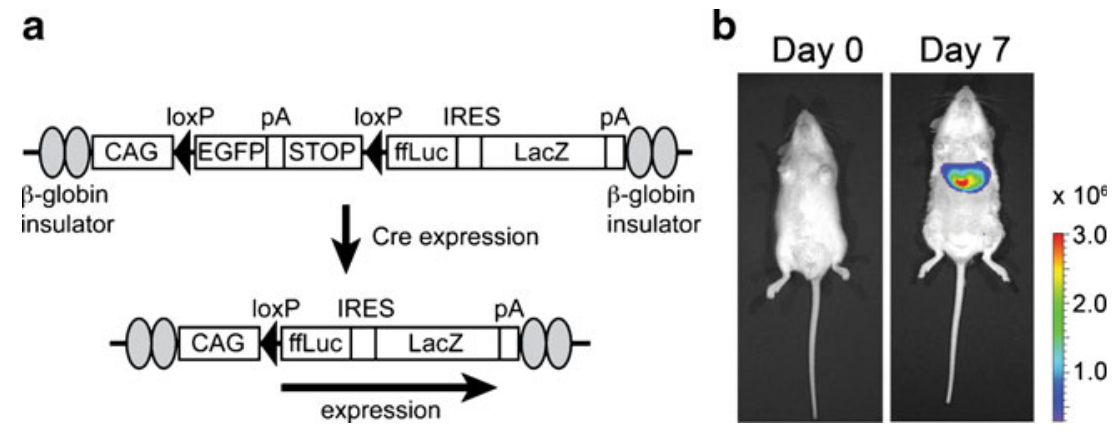

Fig. 1. a) The construct used to create the bicistronic Cre reporter mouse. Cre recombinase mediates the recombination of the two loxP sites flanking the enhanced green fluorescent protein and STOP cassettes, resulting in the expression of luciferase and lacZ message from the CAG promoter. b) In vivo imaging of luciferase activity following intravenous administration of Ad. CMV.Cre. The mouse was imaged prior to and 7 days after adenovirus injection. The color overlay on the image illustrates the photons per second emitted. IRES internal ribosomal entry site, ffLuc firefly luciferase coding region, Lac $Z \beta$-galactosidase coding region, $p A$ SV40 polyA. 
ceutical) mixture. Anesthetized mice were injected intraperitoneally with D-luciferin (125 mg/kg; Caliper Life Sciences, Hopkinton, MA, USA) and placed in the light-tight box of the IVIS 100 optical imaging system (Caliper Life Sciences). Whole body 1-min images were acquired repeatedly until the maximum peak of photon number was confirmed during the 1-min scans. Data at the time point that gave the highest photon number during $1 \mathrm{~min}$ of scanning time were used for quantification. Collected photon number and images were analyzed using LIVING IMAGE software (Caliper Life Sciences).

For ex vivo imaging of isolated tissues, mice were anesthetized and injected with D-luciferin. After the photon accumulation at 1min intervals, examined by in vivo imaging, reached a maximum level, the mice were sacrificed, and the tissues were rapidly excised. Tissues were imaged with the IVIS system.

\section{In vitro Luciferase and $\beta$-galactosidase Assay}

Each tissue was homogenized in passive lysis buffer (Promega). Lysates were centrifuged, and the supernatants were assayed with the luciferase assay system (Promega) and $\beta$-galactosidase enzyme assay system (Promega).

\section{LacZ Histochemical Staining}

Embryos and tissues were fixed in 4\% paraformaldehyde, washed with $\mathrm{PBS}$, and stained in $0.5 \mathrm{mg} / \mathrm{ml} \mathrm{X}$-gal, $5 \mathrm{mM}$ potassium ferrocyanide, and $5 \mathrm{mM}$ potassium ferricyanide in PBS at $30^{\circ} \mathrm{C}$.

\section{LacZ and Luciferase Immunohistochemical Staining}

Embryos were fixed in 4\% paraformaldehyde, paraffin-embedded, and sectioned at $4-\mu \mathrm{m}$ thickness. LacZ expression was detected with rabbit polyclonal anti- $\beta$-galactosidase antibody (Fitzgerald Industries International, Concord, MA, USA) and ImmPRESS antirabbit Ig reagent (Vector Laboratories, Burlingame, CA, USA). To detect luciferase, goat polyclonal antiluciferase (Promega) was used. Signal was visualized by biotinylated rabbit anti-goat IgG and the $\mathrm{ABC}$ reagent kit (Vector laboratories).

\section{Results}

To monitor Cre expression patterns in vivo, we constructed a transgenic mouse line in which a loxP-EGFP-STOP-loxP cassette was placed between a strong, nearly ubiquitous $\mathrm{CAG}$ promoter and a bicistronic reporter gene cassette (Fig. 1a). The firefly luciferase and $l a c Z$ reporter genes were used in the bicistronic transcriptional unit, connected by an ECMV IRES, to facilitate both noninvasive (luciferase) and ex vivo histologic ( $\beta$-galactosidase) examination. The entire transgene is flanked by tandem copies of the chicken $\beta$-globin $5^{\prime}$ HS4 insulator elements, to reduce chromosomal positional effects on gene expression [20, 21]. Transgenic mice were generated by pronuclear injection, and founder lines were selected by genomic Southern blotting and EGFP expression of various tissues. Two founder lines that demonstrated widespread, nearly ubiquitous EGFP expression from the CAG promoter (Fig. 2) were further analyzed for luciferase expression following intravenous injection of Ad.CMV.Cre, an adenovirus that expresses Cre recombinase from the human CMV minimal promoter. Systemically administered adenovirus overwhelmingly infects liver [22, 23]. Both founder lines showed significant luciferase hepatic expression 7 days after Ad.CMV.Cre administration, when assayed noninvasively for luciferase expression (Fig. 1b). For subsequent experiments, we used the founder line that shows both greater luciferase activity and lower basal expression.

Mice transgenic for the Cre recombinase gene driven by a CMV promoter have previously been reported to express Cre in the germ line, as well as in nearly all tissues [24]. To examine consequences of germ line Cre expression and consequent global deletion of the floxed STOP sequence in various tissues of our reporter mouse by noninvasive luciferase bioluminescence, $\beta$-galactosidase histochemistry, and quantitative in vitro luciferase and $\beta$-galactosidase enzymatic activity, the bicistronic luc-gal ${ }^{\mathrm{Tg}}$ reporter mouse was crossed with a CMVCre transgenic mouse. The double transgenic mouse was generated and then crossed with a wildtype mouse, to obtain luc-gal ${ }^{\operatorname{Tg}(\mathrm{del})}$ mice in which the EGFP/ STOP sequence is excised from all cells. The luc-gal ${ }^{\text {Tg(del })}$ mice were examined initially for CAG-directed luciferase expression by noninvasive bioluminescent in vivo imaging (Fig. 3a). Because the pigmented hair of the mouse attenuates the bioluminescent signal from the murine tissues and organs, portions of the mouse body were shaved. After luciferin injection, strong luciferase signal is detectable from shaved areas of both the dorsal and ventral surfaces of the mouse.

Luc-gal $^{\text {Tg(del) }}$ mice, in which the EGFP-STOP sequence between the CAG promoter and the bicistronic reporter gene transcription unit was deleted in all cells, were then sacrificed, and organs were removed, and luciferin-dependent bioluminescence was imaged (Fig. 3b). Extracts were then prepared from the various tissues of the luc-gal ${ }^{\text {Tg(del) }}$ mice, and luciferase and $\beta$-galactosidase enzymatic activities were quantitatively assayed in the tissue lysates. Luciferase and $\beta$ galactosidase activities are observed in all tissues examined (Fig. 3c, d). The level of luciferase activity and the level of $\beta$ galactosidase activity from the CAG promoter vary substantially in the various murine tissues (Fig. 3c, d). However, these data demonstrate that both the noninvasive luciferase reporter and the conventional in vitro $\beta$-galactosidase reporter can indicate Cre recombinase activity in essentially all tissues.

Although substantial increases in the level of activity for both reporters can be detected in all tissues, the ratio of luciferase activity to $\beta$-galactosidase activity in tissue extracts is strikingly different in the various murine tissues (Fig. 3e). The potential reasons for these differences in luciferase/ $\beta$-galactosidase ratios and their implications for use of transgenic reporter mice are considered more extensively in the "Discussion" section. 

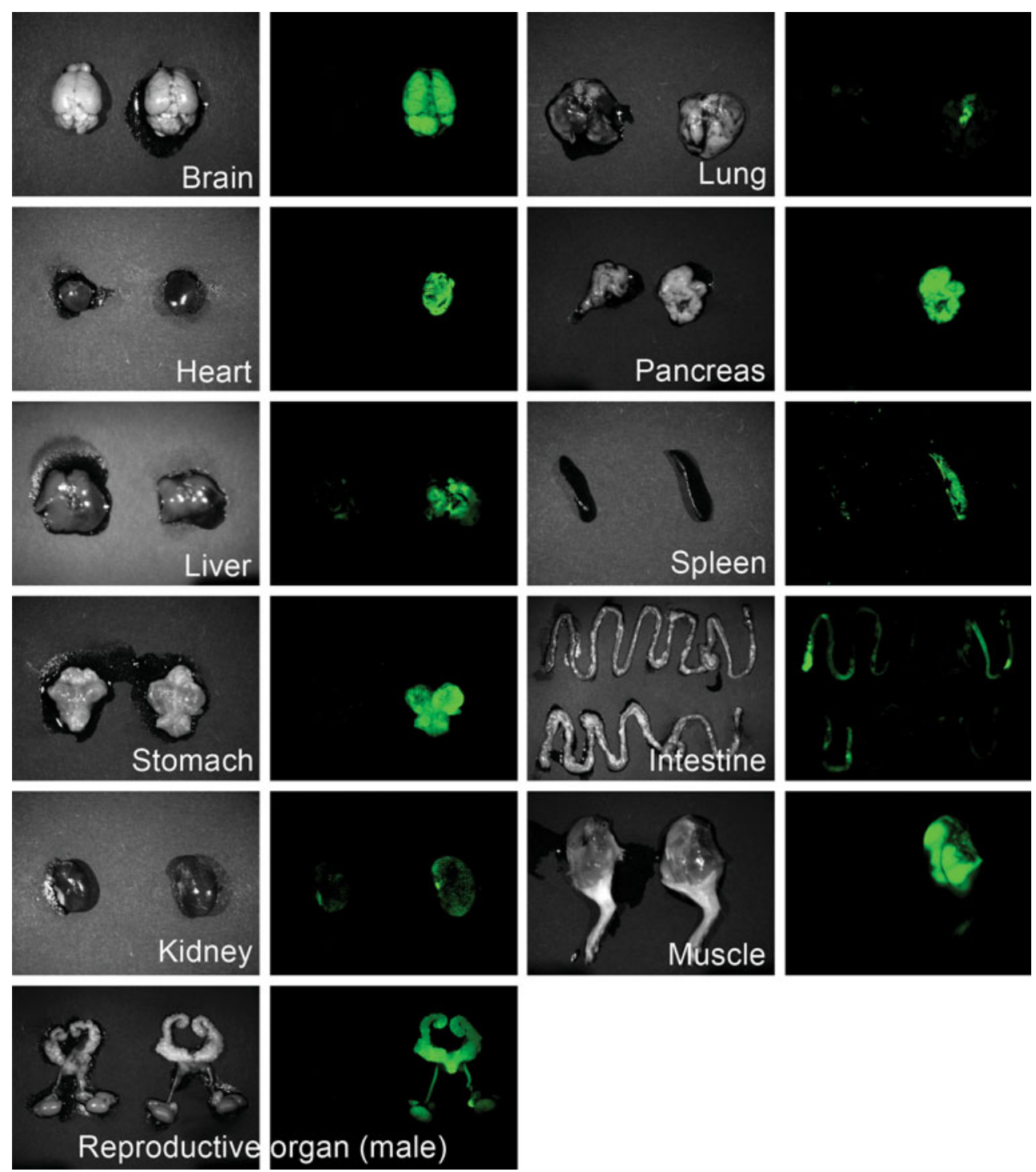

Fig. 2. Enhanced green fluorescent protein fluorescence from organs and tissues from the control wild-type littermate mouse

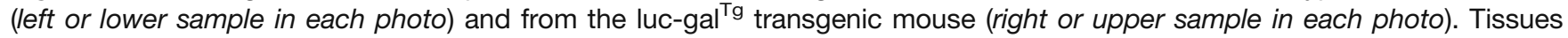
were imaged in the Maestro imaging instrument, and specific signal was extracted by Maestro imaging software.

To demonstrate the utility of the $l a c Z$ reporter gene in analyzing tissue-specific expression, 10.5-day embryos derived from luc-gal ${ }^{\mathrm{Tg}(\mathrm{del})}$ and wild-type mice were stained for $\beta$ galactosidase activity as whole mounts (Fig. 3f). Embryos which have a Cre-activated reporter gene cassette show the characteristic blue color associated with $\beta$-galactosidase activity. In contrast, control embryos do not show any basal staining.

Finally, to demonstrate the advantage of having both luciferase and $\beta$-galactosidase as markers, sections of embryos from Luc-gat ${ }^{\mathrm{Tg}(\mathrm{del})}$ and wild-type littermate mice were stained with either anti- $\beta$-galactosidase antibody or anti-luciferase antibody. While robust staining was confined to the luc-gal ${ }^{\mathrm{Tg}(\mathrm{del})}$ embryo using anti- $\beta$-galactosidase antibody, we were unable to obtain specific staining with antiluciferase antibody (Fig. 4).

To determine whether the luc-gal ${ }^{\mathrm{Tg}}$ reporter line is suitable for detection of tissue-restricted Cre recombinase activity, luc-gal $^{\mathrm{Tg}}$ transgenic mice were crossed with a series of mice that express Cre recombinase from different promoters. The luc-gal ${ }^{\mathrm{Tg}}$ reporter mouse was first crossed with a MlcCre knock-in mouse in which Cre is under the control of the cardiac-specific myosin light chain $2 \mathrm{v}$ promoter [25]. In the luc-gal ${ }^{\mathrm{Tg}} / \mathrm{MlcCre}$ knock-in mouse (luc-gal $^{\mathrm{Tg}} / \mathrm{Mlc}{ }^{\mathrm{Cre} /+}$ ), strong luciferase signal is detected exclusively from the chest region by in vivo imaging (Fig. 5a). In contrast, no luciferase activity is detected from the luc-gal ${ }^{\mathrm{Tg}}$ littermate control that contains the bicistronic reporter gene, but not the gene for Mlc-driven Cre recombinase. After peak overall bioluminescence was observed, the double transgenic luc-gal ${ }^{\mathrm{Tg}} / \mathrm{Mlc}^{\mathrm{Cre} /+}$ mouse and control single transgenic luc-gal ${ }^{\mathrm{Tg}}$ reporter mouse were sacrificed, and organs were removed and imaged (Fig. 5b). Ex vivo luciferase imaging confirms that the bioluminescent signal seen in the noninvasive imaging of the living luc-gal ${ }^{\mathrm{Tg}}$ / 
a
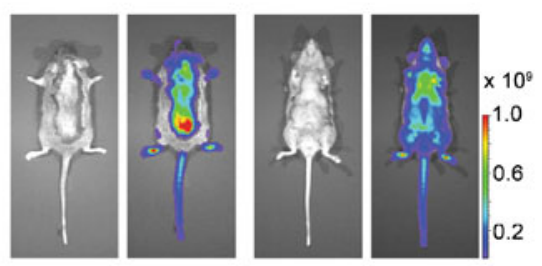

C

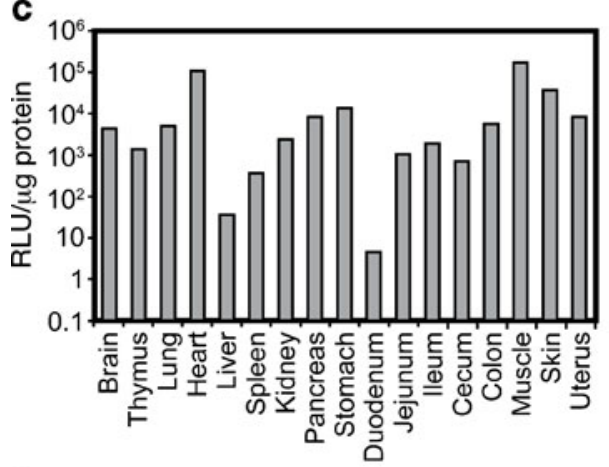

e

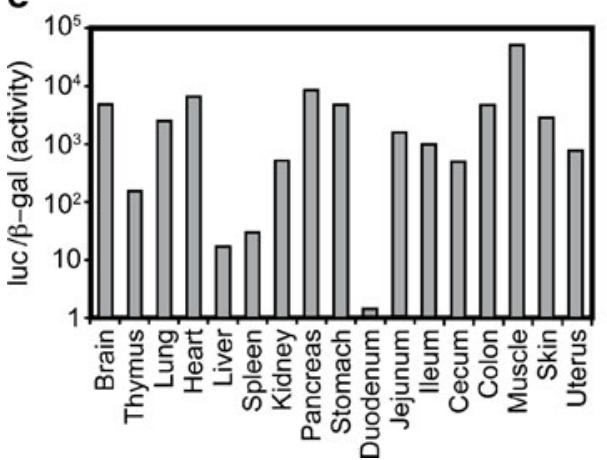

b
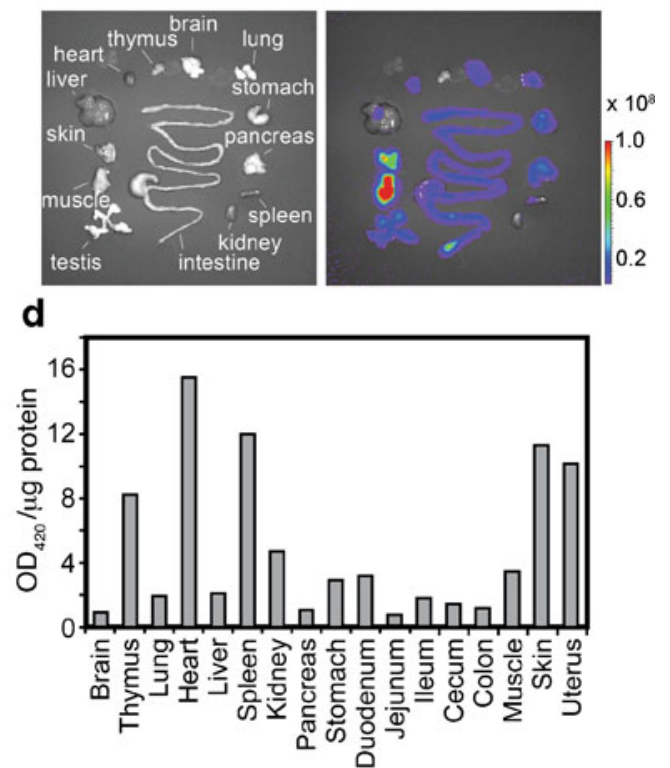

f

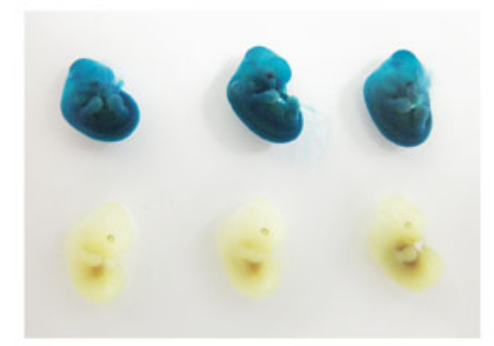

Fig. 3. Reporter gene expression from luc-gal ${ }^{T g(d e l)}$ mice, in which the STOP cassette has been deleted by germline expression of Cre driven by the cytomegalovirus promoter. a) In vivo luciferase imaging. Photos and the color overlays illustrating the photon number are shown for dorsal and ventral images. Note that part of the mouse body is shaved. b) Ex vivo images of luciferase expression in tissues from same mouse shown in (a). c) Luciferase activity in individual tissues measured by in vitro luciferase assay of tissue extracts from luc-gal ${ }^{T(d e l)}$ mice. d) $\beta$-galactosidase activity in individual tissues measured by in vitro $\beta$-galactosidase enzyme assay of the same tissue extracts used in (b). e) Ratio of luciferase/ $\beta$-galactosidase activity for individual tissues in luc-gal ${ }^{\text {Tg(del) }}$ mice. f) Whole mount staining for $\beta$-galactosidase activity of embryos derived from crossing between double transgenic and wild-type mice. The upper row shows embryos containing the luc-gal ${ }^{\mathrm{T} g(d e l)}$ transgene; the lower row shows embryos without the transgene.

$\mathrm{Mlc}^{\mathrm{Cre} /+}$ mouse (Fig. 5a) is from the heart (Fig. 5b). No luciferase signal could be detected from any other organ. When isolated hearts were subsequently stained for $\beta$ galactosidase activity (Fig. 5c), only luc-gal ${ }^{\mathrm{Tg}} / \mathrm{Mlc}^{\mathrm{Cre} /+}$ double transgenic hearts are stained.

The luc-gal ${ }^{\mathrm{Tg}}$ mouse was next crossed with a $\mathrm{K} 14 \mathrm{Cre}$ transgenic mouse. Cre is expressed from the skin epithelial cell-specific keratin 14 promoter in the K14Cre transgenic mouse [26]. In the doubly transgenic luc-gal ${ }^{\mathrm{Tg}} / \mathrm{K} 14 \mathrm{Cre}^{\mathrm{Tg}}$ mouse, luciferase signal is detected on all shaved surfaces of the living animal when analyzed by noninvasive bioluminescent imaging (Fig. 6a). Ex vivo bioluminescent imaging of isolated tissues and organs shows that the signal observed by imaging of the living luc-gal ${ }^{\mathrm{Tg}} / \mathrm{K} 14 \mathrm{Cre}^{\mathrm{Tg}}$ mouse (Fig. 6a) is derived only from skin, and not from internal organs
(Fig. 6b). Skin from the luc-gal ${ }^{\mathrm{Tg}} / \mathrm{K} 14 \mathrm{Cre}^{\mathrm{Tg}}$ mouse was also positive for $\beta$-galactosidase activity (Fig. 6c).

Finally, the luc-gal ${ }^{\mathrm{Tg}}$ mouse was crossed with a VillinCre transgenic mouse. Cre is expressed from the $12.4-\mathrm{kb}$ region of the mouse Villin promoter in the VillinCre transgenic mouse. This mouse is reported to express Cre at high levels within the entire intestinal epithelium [27]. In the doubly transgenic luc-gal ${ }^{\mathrm{Tg}} / \mathrm{VillinCre}{ }^{\mathrm{Tg}}$ mouse, luciferase signal is detected in the abdominal region of the living animal by in vivo imaging, using the IVIS noninvasive optical imaging system (Fig. 7a). Ex vivo optical imaging of isolated organs confirms that the bioluminescence observed in the living luc-gal ${ }^{\mathrm{Tg}} / \mathrm{VillinCre}^{\mathrm{Tg}}$ animal (Fig. 7a) is derived from luciferase expression in the intestine (Fig. 7b). A closer look at the opened colon demonstrates regions of differing 


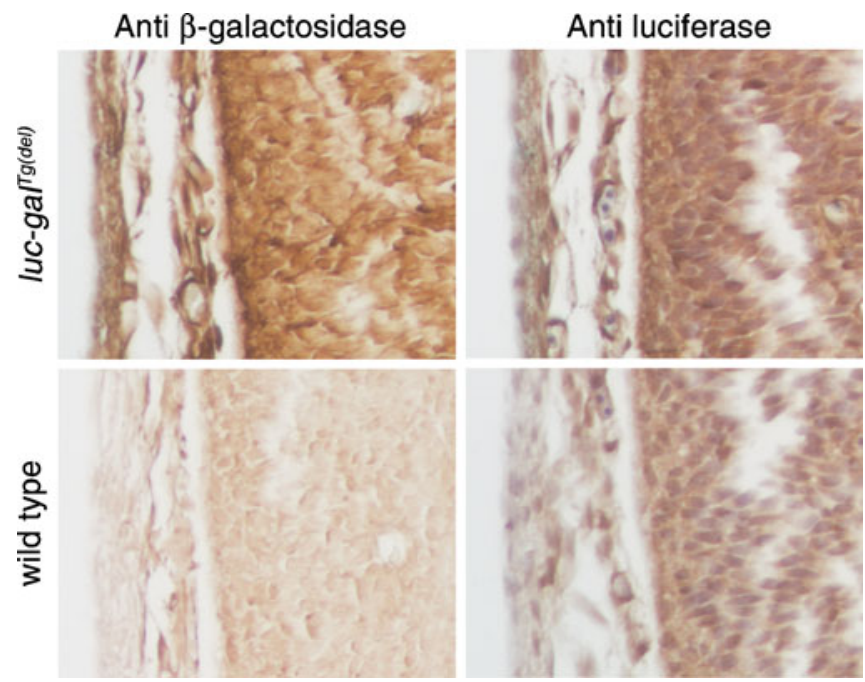

Fig. 4. Immunohistochemical staining of luc-gal ${ }^{T g(d e l)}$ (upper panels) and wild-type littermate (lower pane/s) embryo paraffin sections with anti- $\beta$-galactosidase (left panels) and antiluciferase (right panels) antibodies. A portion of the head region is shown in each section.

levels of luciferase activity in the colon (Fig. 7c). In contrast, no luciferase activity is detectable by bioluminescent imaging of the opened colon of the luc-gal ${ }^{\mathrm{Tg}}$ littermate mouse in which Cre recombinase is not expressed. Moderate luciferase activity is also detected in testis (Fig. 7b), consistent with the report of villin expression in seminiferous ducts [28]. Significant $\beta$-galactosidase activity is also observed in the luc-gal ${ }^{\mathrm{Tg}} / \mathrm{VillinCre}^{\mathrm{Tg}}$ double transgenic mouse intestine, but not in the intestine of the luc-gal ${ }^{\mathrm{Tg}}$ littermate that does not express the VillinCre transgene (Fig. 7d).

\section{Discussion}

In this study, we generated a reporter mouse line in which the luciferase and lacZ reporter gene coding regions are tandemly expressed in a bicistronic complex only when Cre recombinase has removed a STOP sequence between the $\mathrm{CAG}$ promoter and the reporter gene coding regions. The
CAG promoter is an artificial, chimeric promoter that includes portions of the chicken $\beta$-actin promoter and the CMV immediate early enhancer [19]. This promoter is highly active in a wide range of cell types; transgenic mice expressing EGFP from the CAG promoter are reported to show ubiquitous expression and were referred to as "green mice" [29]. Because the luc-gal ${ }^{\mathrm{Tg}}$ reporter mouse described here also has the EGFP reporter gene coding region adjacent to the CAG promoter, we can compare global CAG promoter activity of our mouse to that of the "green mice" originally described by Okabe et al. [29]. Like the original "green mice," we find that essentially all tissues in luc-gal ${ }^{\mathrm{Tg}}$ mice express the EGFP reporter gene from the CAG promoter.

By placing EGFP proximal to the STOP sequence, we have created a mouse in which all tissues can be imaged with EGFP. The penetrance of Cre excision can be estimated, at both the tissue and cellular level, by loss of EGFP expression. Because the luciferase and $\beta$-galactosidase reporters are placed distal to the floxed region of the luc-gal ${ }^{\mathrm{Tg}}$ transgene, selected tissues can be imaged following tissue-specific Cre recombinase deletion of the STOP sequence. Thus, the luc-gal ${ }^{\mathrm{Tg}}$ mouse provides a very flexible reporter mouse from which any tissue or cell expressing the EGFP reporter gene can be isolated and subsequently monitored, either in culture or following transplantation. Moreover, by subsequently introducing Cre recombinase into the luc-gal ${ }^{\mathrm{Tg}}$ cells, a reporter gene that permits more quantitative in vivo evaluation (luciferase), reporter genes that permit more quantitative evaluation in cell extracts (luciferase and $\beta$ galactosidase) and a reporter gene that provides simpler, more sensitive and reliable histochemistry and immunohistochemistry ( $\beta$-galactosidase) are expressed. The combination of three reporters in the luc-gal ${ }^{\mathrm{Tg}}$ mouse gives this system multiple alternatives to evaluate Cre expression.

To verify ubiquitous expression of luciferase and $\beta$ galactosidase in all tissues from the CAG promoter, the loxP-flanked STOP sequence was removed from all cells by deleting the STOP sequence in the germ line and analyzing the progeny for luciferase and $\beta$-galactosidase activity. All organs in the resulting luc-gal ${ }^{\mathrm{T}(\mathrm{del})}$ mouse exhibit elevated
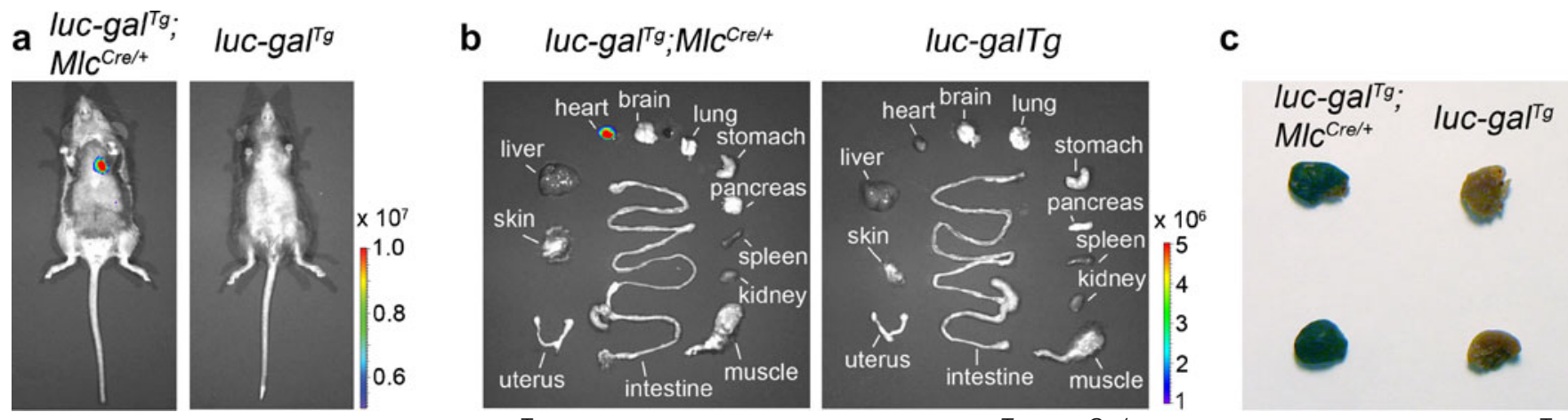

Fig. 5. a) In vivo imaging of the luc-gal ${ }^{T g}$ transgenic/MlcCre knock-in (luc-gal $\left.{ }^{T g} ; \mathrm{Mlc}^{\mathrm{Cre} /+}\right)$ mouse and the control luc-gal ${ }^{T g}$ reporter mouse $\left(l u c-g a l^{T g}\right)$. b) Ex vivo images of luciferase expression in tissues from the same mice shown in (a). c) Whole mount staining for $\beta$-galactosidase activity of hearts from mice shown in (a) and (b). 
a

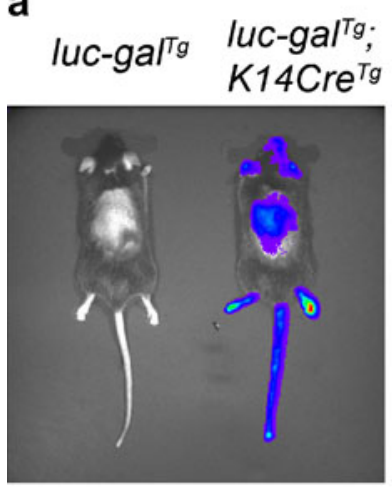

b

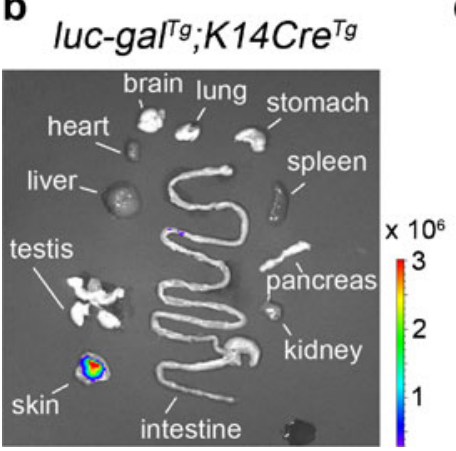

Fig. 6. a) In vivo imaging of the double transgenic luc-gal ${ }^{\top \mathrm{g}}$ reporter/K14Cre ${ }^{T g}\left(\right.$ (luc-gal $\left.{ }^{T g} ; K_{14 C r e}{ }^{T g}\right)$ mouse and the control luc-gal ${ }^{T g}$ reporter mouse (luc-gal ${ }^{T g}$ ). Dorsal and ventral images are shown. Note that part of the mouse body is shaved. b) Ex vivo image of luciferase expression in tissues of double transgenic mouse shown in (a). c) Whole mount staining for $\beta$-galactosidase activity of skin from double transgenic mouse (luc-gal ${ }^{T g} ; K_{14 C r e}{ }^{T g}$ ) and control mouse $\left(\right.$ (luc-gal $\left.{ }^{T g}\right)$.

luciferase and $\beta$-galactosidase activity relative to organs of the luc-gal ${ }^{\mathrm{Tg}}$ mouse (in which the floxed STOP sequence has not been eliminated). However, the level of luciferase expression varies over four orders of magnitude among different organs. Luciferase activity is highest in heart and muscle and lower in intestine and liver, an organ expression profile similar to that of CAG-driven EGFP in the transgenic mouse described by Hadjantonakis et al. [30]. We think these differences in luciferase tissue expression are likely to be due to differences in the efficacy of the CAG promoter in various tissues, rather than to chromosomal positional effects from the transgene integration site, since we flanked the transgene reporter construct by insulator sequences to reduce the effect of integration sites. The ability to quantify accurately the levels of luciferase enzyme activity in tissue extracts and compare these results with the semiquantitative data obtainable with EGFP fluorescence, histochemistry, or western blotting illustrates the flexibility and advantages of the three reporter luc-gal ${ }^{\mathrm{Tg}}$ mouse.

The luciferase reporter provides a sensitive signal with a wide dynamic range and low background. Particularly in its application as a Cre reporter mouse, this combination of luciferase traits-sensitive and semiquantitative analysis in vivo in living animals and excised tissues as well as sensitive and easily quantifiable analysis in extracts - provides an excellent means to establish the qualitative and quantitative degree of Cre excision in in vivo/in vitro experiments. Confirmation of tissue specificity is, of course, important in characterization of murine Cre lines. As cell-specific Cre transgenic and knock-in mouse lines become increasingly available, careful and thorough characterization of the specificity - or lack thereof - of Cre expression becomes increasingly important. Luciferase expression after Cre excision in the luc-gal ${ }^{\mathrm{Tg}}$ mouse is strong enough to be seen clearly by ex vivo imaging even if the Cre expression takes place in only a part of the organ, e.g., VillinCre expression in seminiferous ducts [28]. This strategy should be useful in identifying previously undiscovered promoter activities in unexpected tissues and will be increasingly valuable as additional Cre knock-in strains, in which Cre expression should more accurately reflect the promoter activity of the targeted gene, are developed.

The luciferase reporter has a major advantage over fluorescent reporter proteins; although fluorescent proteins

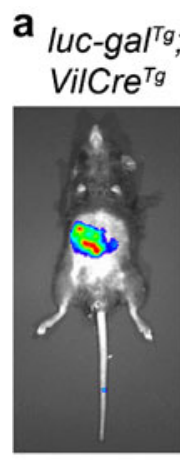

c

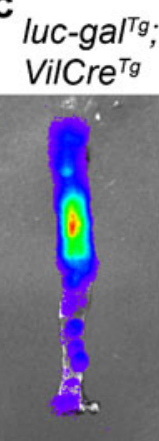

b

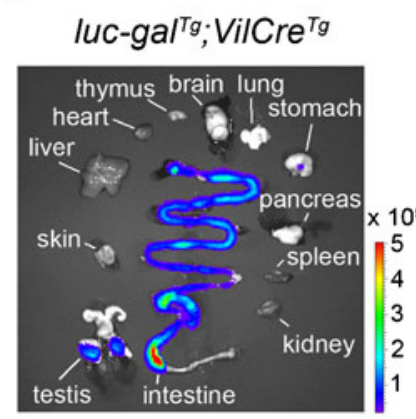

d

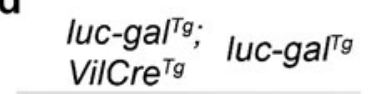

Fig. 7. a) In vivo imaging of the double transgenic luc-gal ${ }^{T g}$ reporter/VillinCre ${ }^{T g}$ (luc-gal $\left.{ }^{T g} ; V_{i l C r e}{ }^{T g}\right)$ mouse and the control luc-gal ${ }^{T g}$ reporter mouse (luc-gal ${ }^{T g}$ ). b) Ex vivo image of luciferase expression in tissues of the luc-gal ${ }^{T g} / \mathrm{NillinCre}^{\mathrm{Tg}}$ double transgenic mouse shown in (a). c) Ex vivo images of luciferase expression in opened colons of the luc-gal $\left.\right|^{\mathrm{Tg}}$ / VillinCre ${ }^{T g}$ double transgenic (luc-gal ${ }^{T g}$; VilCre ${ }^{T g}$ ) mouse and the control luc-gal ${ }^{T g}$ reporter mouse (luc-gal ${ }^{T g}$ ) shown in (a). d) Whole mount staining for $\beta$-galactosidase activity of duodenum from a double transgenic luc-gal ${ }^{\mathrm{Tg}}$ reporter/ VillinCre ${ }^{T g}$ (luc-gal ${ }^{T g} ; V_{i l C r e}{ }^{T g}$ ) mouse and a control luc$\mathrm{gal}^{\mathrm{Tg}}$ reporter (luc-gal ${ }^{T g}$ ) mouse. 
can be imaged in living mice, it is often difficult to distinguish the reporter gene signal from tissue autofluorescence, particularly from internal organs. While both bioluminescent proteins such as luciferase and fluorescent proteins such as EGFP suffer from scattering and absorption that reduce their resolution and sensitivity in vivo, luciferase measurements are not compromised by endogenous light emission. Thus, detection of specific signals from internal organs such as heart and intestine is more easily accomplished with the luciferase reporter. Luciferase imaging in the luc-gat ${ }^{\mathrm{Tg}}$ mouse may also provide some opportunities for longitudinal monitoring of cell lineage and cell movement in living animals, in addition to the evaluation of the specificity of Cre recombinase expression. For more quantitative in vivo imaging in living animals, reporter genes that use technologies such as positron emission tomography need to be employed [31-33].

The major disadvantage of luciferase as a reporter gene is at the cellular level. Antisera that provide reliable, easy, and reproducible immunohistochemistry for luciferase are not available. We have compensated for this deficiency by utilizing lacZ, another reporter gene, in tandem with luciferase to monitor tissue-specific Cre recombinase activity at the organ, tissue, and cellular level by histochemical and immunohistochemical detection methods, both on sections and on whole mount samples. Although reports of immunohistochemical detection of luciferase activity following injection of luciferase-encoding plasmids into tissues do exist $[34,35]$, we find that the far more robust immunohistochemistry methods available for $\beta$-galactosidase $[36,37]$ make cellular analysis of transgene expression much simpler to interpret.

Although the same luciferase/ECMV IRES/ $\beta$-gal transcript is expressed in all tissues of the luc-gal ${ }^{\mathrm{Tg}}$ mouse following Cre recombinase removal of the STOP sequence, the luc/ $\beta$-galactosidase activity ratio varies quite substantially among different organs. At least three possibilities exist to explain this variability: (1) There may exist codons in these non-mammalian proteins that are translated more effectively in one cell type relative to another, e.g., as a result of different tRNA expression levels in different tissues. (2) Efficacy of assembly of the $\beta$-galactosidase tetramer may differ from tissue to tissue. (3) The more likely explanation for the difference in the luc/ $\beta$-galactosidase ratio in different tissues is a tissue-specific difference in the ability of various cell types to initiate translation from the $\beta$ galactosidase coding unit distal to the IRES. Although the location of the $\beta$-galactosidase coding unit distal to the IRES undoubtedly reduces the sensitivity of this reporter gene, the well-characterized, sensitive, robust, and widely used in vitro enzyme activity assays, histochemical assays, and immunohistochemistry reagents and protocols for $\beta$-galactosidase $[36,37]$ suggest that this compromise will not be a practical impediment to employing the various $\beta$-galactosidase reporter modalities of the luc-gal ${ }^{\mathrm{Tg}}$ mouse. Indeed, this mouse may serve as a useful tool in understanding the properties and functions of IRES.
A variety of mouse reporter lines to monitor Cre activity has been generated previously. Each line contains different reporter genes and has consequent advantages and disadvantages. The luc-gal ${ }^{\mathrm{Tg}}$ reporter mouse, with EGFP, luciferase, and $\beta$-galactosidase reporter genes that can be used in various combinations and applications, should provide an additional valuable and useful mouse with which to evaluate Cre recombinase activity and to monitor cell engraftment, proliferation, movement, and differentiation in a number of contexts.

Acknowledgements. We thank Art Catapang for technical assistance. These studies were supported by grants R01 CA84572 and P50 CA86306 from the National Cancer Institute to HRH.

Open Access. This article is distributed under the terms of the Creative Commons Attribution Noncommercial License which permits any noncommercial use, distribution, and reproduction in any medium, provided the original author(s) and source are credited.

\section{References}

1. Nagy A (2000) Cre recombinase: the universal reagent for genome tailoring. Genesis 26:99-109

2. Feil R (2007) Conditional somatic mutagenesis in the mouse using sitespecific recombinases. Handb Exp Pharmacol 178:3-28

3. Mao X, Fujiwara Y, Orkin SH (1999) Improved reporter strain for monitoring Cre recombinase-mediated DNA excisions in mice. Proc Natl Acad Sci USA 96:5037-5042

4. Lobe CG, Koop KE, Kreppner W et al (1999) Z/AP, a double reporter for cre-mediated recombination. Dev Biol 208:281-292

5. Yamauchi Y, Abe K, Mantani A et al (1999) A novel transgenic technique that allows specific marking of the neural crest cell lineage in mice. Dev Biol 212:191-203

6. Soriano P (1999) Generalized lacZ expression with the ROSA26 Cre reporter strain. Nat Genet 21:70-71

7. Novak A, Guo C, Yang W et al (2000) Z/EG, a double reporter mouse line that expresses enhanced green fluorescent protein upon Cremediated excision. Genesis 28:147-155

8. Kawamoto S, Niwa H, Tashiro F et al (2000) A novel reporter mouse strain that expresses enhanced green fluorescent protein upon Cremediated recombination. FEBS Lett 470:263-268

9. Srinivas S, Watanabe T, Lin CS et al (2001) Cre reporter strains produced by targeted insertion of EYFP and ECFP into the ROSA26 locus. BMC Dev Biol 1:4

10. Mao X, Fujiwara Y, Chapdelaine A et al (2001) Activation of EGFP expression by Cre-mediated excision in a new ROSA26 reporter mouse strain. Blood 97:324-326

11. Vintersten K, Monetti C, Gertsenstein M et al (2004) Mouse in red: red fluorescent protein expression in mouse ES cells, embryos, and adult animals. Genesis 40:241-246

12. Luche H, Weber O, Nageswara RT et al (2007) Faithful activation of an extra-bright red fluorescent protein in "knock-in" Cre-reporter mice ideally suited for lineage tracing studies. Eur J Immunol 37:43-53

13. Muzumdar MD, Tasic B, Miyamichi K et al (2007) A global doublefluorescent Cre reporter mouse. Genesis 45:593-605

14. De Gasperi R, Rocher AB, Sosa MA et al (2008) The IRG mouse: a two-color fluorescent reporter for assessing Cre-mediated recombination and imaging complex cellular relationships in situ. Genesis 46:308-317

15. Lyons SK, Meuwissen R, Krimpenfort P et al (2003) The generation of a conditional reporter that enables bioluminescence imaging of Cre/ loxP-dependent tumorigenesis in mice. Cancer Res 63:7042-7046

16. Safran M, Kim WY, Kung AL et al (2003) Mouse reporter strain for noninvasive bioluminescent imaging of cells that have undergone Cremediated recombination. Mol Imaging 2:297-302

17. Herschman HR (2003) Molecular Imaging: looking at problems, seeing solutions. Science 302:605-608

18. Contag PR, Olum IN, Stevenson DK et al (1998) Bioluminescent indicators in living mammals. Nat Med 4:245

19. Niwa H, Yamamura K, Miyazaki J (1991) Efficient selection for highexpression transfectants with a novel eukaryotic vector. Gene 108:193-199 
20. Potts W, Tucker D, Wood H et al (2000) Chicken beta-globin 5'HS4 insulators function to reduce variability in transgenic founder mice. Biochem Biophys Res Commun 273:1015-1018

21. Ciana P, Di Luccio G, Belcredito S et al (2001) Engineering of a mouse for the in vivo profiling of estrogen receptor activity. Mol Endocrinol 15:1104-1113

22. Smith TAG, Mehaffery MG, Kayda DB et al (1993) Adenovirus mediated expression of therapeutic plasma levels of human factor IX in mice. Nat Genet 5:397-402

23. Herz J, Gerard D (1993) Adenovirus-mediated transfer of low density lipoprotein receptor gene acutely accelerates cholesterol clearance in normal mice. Proc Natl Acad Sci USA 90:2812-2816

24. Schwenk F, Baron U, Rajewsky K (1995) A cre-transgenic mouse strain for the ubiquitous deletion of loxP-flanked gene segments including deletion in germ cells. Nucleic Acids Res 23:5080-5081

25. Chen J, Kubalak SW, Minamisawa S et al (1998) Selective requirement of myosin light chain $2 \mathrm{v}$ in embryonic heart function. J Biol Chem 273:1252-1256

26. Dassule HR, Lewis P, Bei M et al (2000) Sonic hedgehog regulates growth and morphogenesis of the tooth. Development 127:4775-4785

27. Madison BB, Dunbar L, Qiao XT et al (2002) Cis elements of the villin gene control expression in restricted domains of the vertical (crypt) and horizontal (duodenum, cecum) axes of the intestine. J Biol Chem 277:33275-33283

28. Horvat B, Osborn M, Damjanov I (1990) Expression of villin in the mouse oviduct and the seminiferous ducts. Histochemistry 93: $661-663$
29. Okabe M, Ikawa M, Kominami K et al (1997) 'Green mice' as a source of ubiquitous green cells. FEBS Lett 407:313-319

30. Hadjantonakis AK, Gertsenstein M, Ikawa M et al (1998) Generating green fluorescent mice by germline transmission of green fluorescent ES cells. Mech Dev 76:79-90

31. Gambhir SS, Bauer E, Black ME et al (2000) A mutant herpes simplex virus type 1 thymidine kinase reporter gene shows improved sensitivity for imaging reporter gene expression with positron emission tomography. Proc Natl Acad Sci USA 97:2785-2790

32. Liang Q, Satyamurthy N, Barrio JR et al (2001) Noninvasive, quantitative imaging in living animals of a mutant dopamine D2 receptor reporter gene in which ligand binding is uncoupled from signal transduction. Gene Ther 8:1490-1498

33. Chung JK (2002) Sodium iodide symporter: its role in nuclear medicine. J Nucl Med 43:1188-1200

34. Ashley WW Jr, Russell B (2000) Tenotomy decreases reporter protein synthesis via the 3 '-untranslated region of the beta-myosin heavy chain mRNA. Am J Physiol Cell Physiol 279:C257-C265

35. Salerno MS, Thomas M, Forbes D et al (2004) Molecular analysis of fiber type-specific expression of murine myostatin promoter. Am J Physiol Cell Physiol 287:C1031-C1040

36. Cui C, Wani MA, Wight D et al (1994) Reporter genes in transgenic mice. Transgenic Res 3:182-194

37. Alam J, Cook JL (1990) Reporter genes: application to the study of mammalian gene transcription. Anal Biochem 188:245-254 